\title{
A New Modeling and Analysing Approach to Material Flow and Productivity - An Application of Manufacturing Systems Theory (MST) to Production Systems
}

\author{
Kesheng Wang \\ Professor, Dr. ing., Department of Production and Quality Engineering \\ NTH, N-7034 Trondheim, Norway \\ Tel: 4773597119 Fax: 4773597117 Email: keshengw@protek.unit.no
}

\begin{abstract}
This paper describes an application of Manufacturing Systems Theory (MST) to the modeling and analysis of material flows and productivity of a batch-processing engineering company which produces a large variety of electrical motors. In MST, the various physical and economical systems within manufacturing engineering may be modeled and analyzed by one abstract geometrical system. The transformation from physical variables (concrete) to geometrical variables (abstract) has a dramatic reduction on the number of modelling approaches. The modeling approach is more general, systematic, unified and effective than any other approach.
\end{abstract}

\section{Key words}

Modeling and analysis, graph theory, systems theory, manufacturing systems, production planning and control

\section{Introduction}

Manufacturing engineering is one of the most important fields in the world today. The modeling of manufacturing systems helps in analyzing various designing, planning and operating decisions. In recent years, there has been a rapid growth in interest in the basic theoretical and methodological issues in the modeling and analysis of a variety of complex large-scale systems. The modeling approaches for manufacturing systems reported in literature include linear programming, dynamic mathematical programming, markov method, simulation model, network model, bond graph, queuing model, pertrinets, etc. [4,5,6,7,8,12,13] Most of these approaches are domain-dependent, that means none of them can be suitable to model all kinds of problems in manufacturing systems.

With the increasing technological complexity and scale of manufacturing systems which spans from design, manufacture to management, the request to derive solutions of problems more 
objectively, systematically and logically could never be overemphasized. Theory of Technical system, [9] Physical System Theory, [10] System Science, and Computer aided sy stems theory [11] are all developed for meeting this request.

The Purpose of developing Manufacturing Systems Theory [1,3,15] is also exactly to meet the request for the modeling and analysis of large-scale and complex manufacturing systems. In Manufacturing Systems Theory, the large amount of physical and economical systems within manufacturing engineering may be modelled and analyzed by one geometrical system. All these physical variables within the systems can be interpreted as a set of geometric variables. This transformation leads to a dramatic reduction on the number of modelling approaches.

In this paper we will present how to use a new modeling and analyzing approach, i.e., by Manufacturing Systems Theory (MST), to model and analyze flows of material, cost of unit weight and productivity in a multi-stage production system. The system is a batch-processing engineering which produces a large variety of electrical motors. The approach of modeling and analyzing is described in the following sections in details.

For the reason of comparisons and simplifications, the case study is exactly the same as the one of reference 12 . The production system consists of 12 production stages. There are two production lines; one for manufacturing of rotors and other for stators. These components are finally assembled. A set of standard functions written in APL (A Programming Language) has been used for the automatic computer-aided analysis. A complete program list is proposed for the illustrated example.

\section{Manufacturing Systems Theory}

The general procedure of modeling and analyzing in MST for any kind of system is briefly described as the following :

(1) Set up primitive system (Y-object);

In this stage, a whole system will be disconnected into several logicallyindependent units, which are called elements or primitive systems. The physical variables (dual variables) and govern equations (the relationship of the physical variables) of each element are defined. Then the characteristics of all elements are represented in mathematics by a Y-object.

(2) Set up the connected system (V-object);

The element can be modeled by a directed line and two boundary nodes, and the connection of all these elements can be modeled by a directed linear graph, which is called a behavior graph of a system. The topology of a connected system can be represented as V-object algebraically.

(3) Determine the given source $\left(\mathrm{I}_{\mathrm{N}}\right.$-object);

The influence to a system from environment may be selected as sources.

(4) Solve the problem $\left(e_{N}\right.$-object). 
After the $\mathrm{Y}-, \mathrm{V}-$, and $\mathrm{I}_{\mathrm{N}}$-object has been established, the solution process is just as simple as to transform the primitive systems into the connected system.

$$
\mathbf{e}_{\mathrm{N}}=\mathrm{V}^{\mathrm{t}} \mathbf{Y} \mathbf{V} \mathbf{I}_{\mathrm{N}}
$$

The procedure described above shows that the approach is general, systematic, unified and eifective compared to the other approach.

\section{Modeling of a transformation process}

The different components of a production system under consideration are conceptualized to perform any one, or a combination, of the following basic processes:

(a) Transformation process: the transformation process can be defined as a transformation of resources to achieve a well-defined change in their physical, chemical, technological, biological or functional characteristics.

(b) Transportation process: This is a special type of transformation process in which material is simply moved from one geographic location to another at a cost. This includes collection, translocation and distribution of various kinds of materials.

(c) Storage processes: This is a special type of transformation process in which the input and output are identical in form, and the resources are carried over time.

The MST has been successfully applied to modeling and analysis of the transportation process and storage process.[13] This paper will focus on the material transformation processes taking place within a production system. The free body diagram of the ith transformation process is shown in Fig. 1.

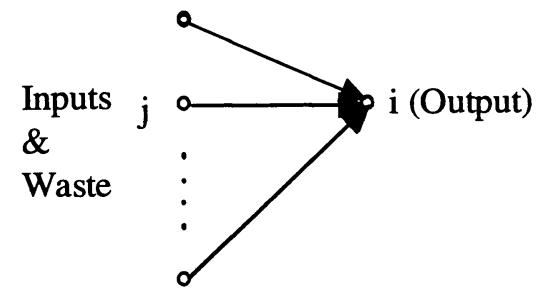

Figure 1. Free body diagram of ith transformation process

The response-stimulus relationships for a transformation process are as follows:

(1) Material flow equation:

$$
I_{j}=Y_{j} I_{i}
$$

(2) Cost equation:

The associated parameters and variables are as follows: 


$$
E_{i}=\Sigma Y_{j} E_{j}+f_{i}\left(I_{i}\right)
$$

$I_{j}=$ flow of material input, or waste, of the jth type,

$\mathrm{I}_{\mathrm{i}}=$ material output, or design capacity, of the process,

$\mathrm{E}_{\mathrm{j}}=$ cost per unit material input, or waste, of the jth type,

$\mathrm{E}_{\mathrm{i}}=$ unit cost of cutput of ith process,

$Y_{j}=$ process technological coefficient for input, or waste, of jth type.

$f_{\mathrm{i}}=$ the cost function, which represents the variation in processing cost with design capacity which accounts for labor, capital and overhead costs by the technological coefficient at various stages.

\section{(3) Sensitivity}

The sensitivity $S_{j}$ for the change in output cost for a corresponding unit change in some factor at any stage can be carried out by partial-differentiating the output cost, $E_{\text {out }}$, with respect to the technological coefficient, $Y_{\text {, }}$ at various stages, as follows:

$$
S_{j}=\frac{\partial E_{\text {out }}}{\partial Y_{j}}
$$

(4) Production index

In the system, productivity $P_{i}$ is defined as the ratio of the ideal output cost per unit weight to the actual cost per unit weight, i.e.,

$$
P_{i}=\frac{\text { ideal cost per unit weight }}{\text { actual cost per unit weight }} \cdot 100
$$

Production index $\mathrm{P}_{\mathrm{i}}$ will help in identifying potential areas of improvement. It is worth nothing that the productivity index for this system will always be less than 100 except in the rare case when it could be equal to 100 . For a fully, or highly, automated industry, a high index number, approaching 100, could be regarded as satisfactory, while in a labour-intensive industry a much lower value could be considered good. The interpretation of these indices will depend on individual managements.

\section{A case study}

\subsection{Description of the production system}

The purpose of the application of MST is to analyze the inter-relationships between the various process of the existing system in order to identify areas of productivity improvement. The given production system consists of 12 production stages. There are two production lines; one for the manufacture of rotors, and the other for stators. These components are finally assembled together into a product. 


\subsection{Primitive systems}

According to the concept of MST, a complete and whole system can always be separated into a number of subsystems and a subsystem may be divided further into several elements which are no longer interconnected each other, i.e., are logically independent. The collection of elements are called primitive systems. The given production system can be viewed as a whole system, the various production stages as subsystems and each transformation process as an element. The present production system consists of 7 subsystems (production stages), i.e., transformation processes, are: (1) cast iron foundry, (2) machining, (3) press working, (4) winding, (5) die casting, (6) balancing, and (7) assembly.

\subsubsection{Elements in primitive systems}

The basic components in the primitive systems are elements. In other words, a primitive system is a physical element which has terminal pairs at which different measurements are made, that is, all measuring procedures (instruments) have, at least conceptually, two terminal points. A measurement therefore always has to be associated with a topological line having two boundary points coinciding with the points in which the instrument is connected. Now let's explain how to use a directed topological line with two boundary nodes to model a transformation process. The first transformation process in the production system, foundry, is considered. In foundry, the flow of material input, Pig iron, is transferred into the flow of material output, raw casting, and the waste or rejects. The foundry process may be viewed as a subsystem, and the subsystem consists of two primitive systems, i.e., two elements. One element is the basic transformation process that pig iron is transformed into raw casting, and another element is one that pig iron is transformed into waste and rejects. Each element is modeled as a directed topological line and nodes represents total flow of material input, output and waste or rejects respectively. The direction of line is based on the requirement of computation. The rule for determining the direction of a arrow is that in the case of computing material flow, the direction of an arrow is against the direction of the transformation process, and in the case of cost calculation, the direction of an arrow is same as the direction of the transformation process. The diagram of the foundry process is shown in figure 2 .

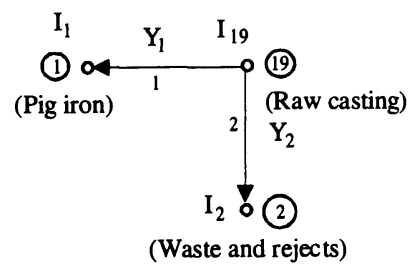

(a)

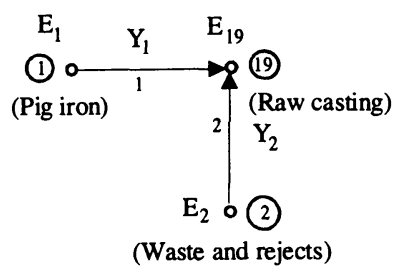

(b)

Figure 2. The diagram of primitive system of the Foundry.

(a) for the flow of material, (b) for the cost analysis.

\subsubsection{Dual variables}

In transformation process system, there are two different kinds of physical quantities to 
characterize the properties of an element : the unit cost of material is defined as a transvariable denoted by $\mathrm{E}$, while the flow of material is defined as a intervariable denoted by I. The technological coefficient representing the input requirement per unit of output is denoted by $\mathrm{Y}$.

\subsubsection{Relationship between the dual variables}

In foundry process, material flow equation are as follows:

$$
\begin{aligned}
& I_{1}=Y_{1} I_{19} \\
& I_{2}=Y_{2} I_{19}
\end{aligned}
$$

and cost equation is:

$$
E_{19}=Y_{1} E_{1}+Y_{2} E_{2}+f_{19}\left(I_{19}\right)
$$

Where

$I_{1}=$ total input of pig iron, $I_{2}=$ total foundry scrap or waste, $I_{19}=$ total output of raw casting, $E_{1}=$ unit cost of pig iron, $E_{2}=$ unit cost of disposing foundry scrap, $E_{19}=$ unit cost of raw casting, $Y_{1}=$ the pig iron required unit of raw casting, $Y_{2}=$ the scrap generated per unit of raw casting.

\subsubsection{Y-object}

All kinds of physical variables are represented with $\mathrm{N}$-object in MST. The representation of $\mathrm{N}$-object is easy to be carried out in a computer. In the present example, Y-object is a 2object, i.e., a diagonal matrix, which may be derived from the diagrams of primitive systems shown in Figure 3. And Y-object may be set up as followings:

$$
\mathbf{Y}=\operatorname{diag}\left[\mathbf{Y}_{1}, \mathbf{Y}_{2}, \ldots . . . \mathbf{Y}_{\mathrm{n}}\right]
$$

where $n=29$ and is the number of the directed lines of the system.

\subsection{Interconnected system}

The important stage in MST is to set up the connection of the individual primitive systems. The complete interconnected system is modeled by a directed linear graph which characterizes the topological property of the system.

\subsubsection{Behavior graph}

The behavior graph for computing material flow is found just by joining the diagram of primitive systems together. In a complete behavior graph, all nodes have to be connected to a common reference. But for simplification, we will never really bring the corresponding lines and the reference points into the behavior graph. We have to keep it in mind that an identity matrix, YI, is always added to a transformed YN-object.[2] The simplified behavior graph is shown in Figure 4. 

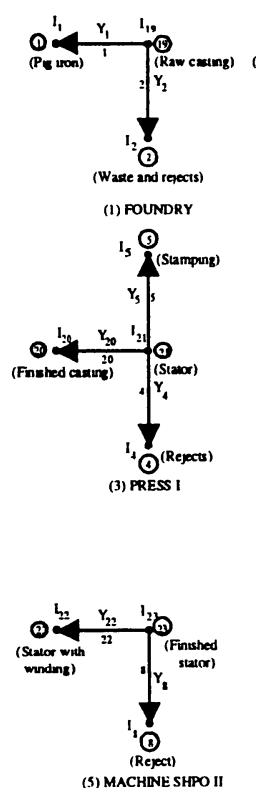
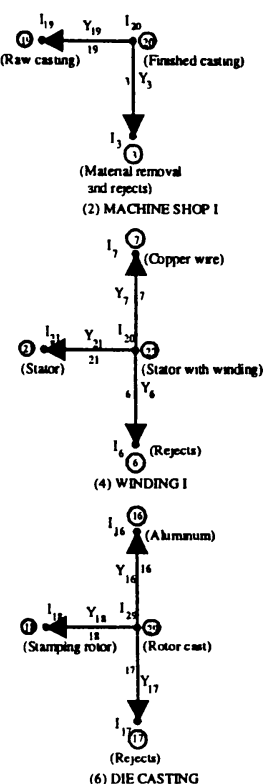
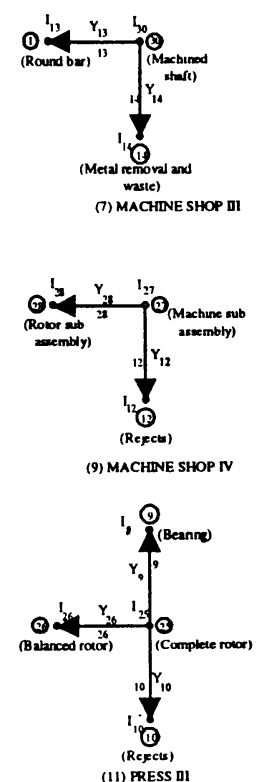

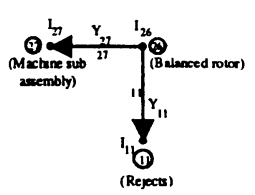

(10) BNLANCTNG

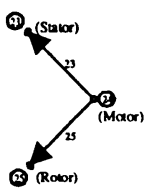

(12) ASSEMBLY

Figure 3. The diagram of primitive systems.

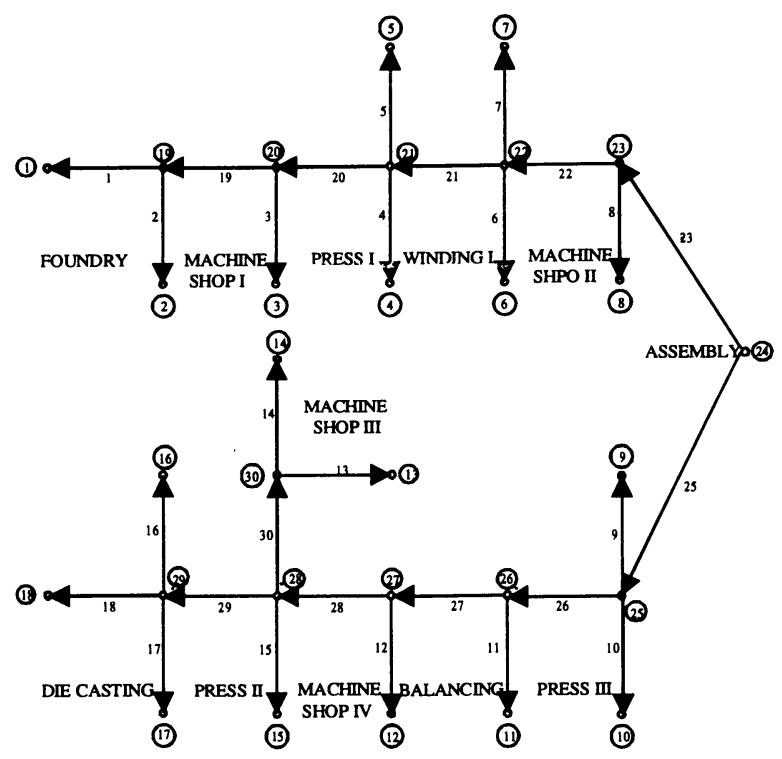

Figure 4. The simplified behavior graph of the production system 
The behavior graph of the production system for cost computation is obtained just by turning the direction of the arrows in the behavior graph for flow of material computation. So we will not show that diagram.

\subsubsection{Topological structure}

The behavior graph above enables us to directly derive the corresponding topological matrices. The total incidence matrix VU, the positive incidence matrix VP, and the negative incidence matrix VN are defined:

$$
\begin{aligned}
& V U_{i j}= \begin{cases}+1 \text { with tail of an arrow } \\
-1 \text { with head of an arrow } \\
0 & (\text { else })\end{cases} \\
& V P_{i j}= \begin{cases}+1 \text { with tail of an arrow } \\
0 & \text { (else) }\end{cases} \\
& V N_{i j}= \begin{cases}+1 \text { with head of an arrow } \\
0 & \text { (else) }\end{cases}
\end{aligned}
$$

where $\mathrm{i}=$ the number of lines and $\mathrm{j}=$ the number of nodes.

\subsection{Given sources}

In MST, sources are defined as the influence between a system and environment. We only need to consider node sources for the production system. There are two kinds of node sources :

(a) Node source for material flow: The analysis of material flow is to help a manager to make the bill of material for producing necessary products. The easiest way is to compute the necessary products for one final product, ie, one motor in the present example. The node source can be represented as a 1-object form (vector):

$$
\left.I_{N}=\begin{array}{rrrrrrrrrr}
1 & 2 & 3 & & 22 & 23 & 24 & 25 & 26 \\
0 & 0 & 0 & \ldots & 0 & 0 & 1 & 0 & 0 & \ldots
\end{array}\right]
$$

The number of elements in the IN is equal to the number of nodes. One motor is produced in the 24th node (see Fig. 4).

(b) Node sources for the cost of unit weight: The data collection mainly consists of the material, labour, capital and overhead costs incurred as well as the quantity of material waste generated at various stages. The data for material cost per unit weight are derived from available accounting data as given as followings:

$$
\begin{aligned}
& E_{1}=1028, \quad E_{2}=-250, \quad E_{3}=-150, \quad E_{4}=-150, \quad E_{5}=13632, \quad E_{6}=-10000, \\
& \mathrm{E}_{7}=40972, \quad \mathrm{E}_{8}=-200, \quad \mathrm{E}_{9}=113119, \quad \mathrm{E}_{10}=-200, \quad \mathrm{E}_{11}=-200, \quad \mathrm{E}_{12}=-200,
\end{aligned}
$$


$E_{13}=332, \quad E_{14}=50, \quad E_{15}=-200, \quad E_{16}=13667, \quad E_{17}=-400 \quad E_{15}=13632$

The minus sign represents that the waste or rejects can be reused.

The average estimated values of the factor $f$ for different stages are given as following:

$$
\begin{array}{ll}
f_{19}=5.32, f_{20}=4.71, f_{21}=0.231, f_{22}=2.81, & f_{23}=0.84, \quad f_{24}=8.63, f_{25}=5.46, \\
f_{26}=0.44, f_{27}=1.84, f_{28}=0.46, \quad f_{29}=0.44, & f_{30}=0.57
\end{array}
$$

The node source $\mathrm{I}_{\mathrm{NC}}$ may be set up based on the data which given above:

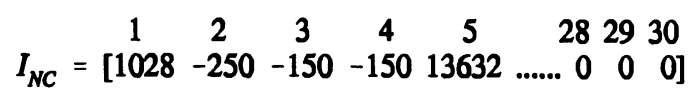

The factor $f$ may be represented as :

$$
\begin{aligned}
& \begin{array}{lllllll}
12 & 34 & 27 & 28 & 29 & 30
\end{array} \\
& f=\left[\begin{array}{lllllllll}
0 & 0 & 0 & 0 & \ldots . . . & 1.84 & 0.44 & 8.63 & 5.46
\end{array}\right]
\end{aligned}
$$

\subsection{Formulation and solution}

\subsubsection{Roth's diagram for ordinal scale of measurement system}

The elements in a production system is characterized by ordering elements which has inherent orientation.[1,2]. The corresponding system is called a system of ordinal scale of measurement. The change of reference frame in the case of ordinal scale of measurement is performed by the positive part of V-object, VP, and the negative part, VN. Roth's diagram in Figure 5 shows the transformation process graphically. All the formulation processes are carried out based on the Roth's diagram routinely.

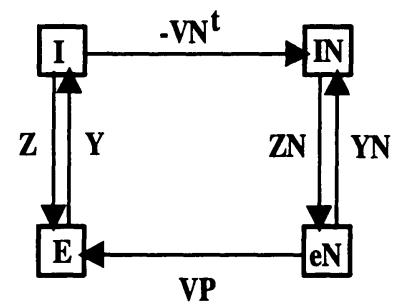

Figure 5. Transformation diagram of the ordinary scale system

\subsubsection{Formulation}

(a) For the computation of material flow, formulation is as the follows

$$
\mathbf{Y N}=-\mathbf{V} \mathbf{N}^{\mathrm{t}} \mathbf{Y} \mathbf{V P}
$$




$$
\begin{aligned}
& \mathbf{Y N N}=\mathbf{Y N}+\mathbf{Y I} \\
& \mathbf{Z N B}=\mathbf{Y N N}^{-1} \\
& \mathbf{e N B}=\mathbf{Z N} \mathbf{I N B}
\end{aligned}
$$

(b) For the cost calculation, formulation become

$$
\begin{aligned}
& \text { ZNC }=\text { ZNB }^{\text {t }} \\
& \text { F }=\text { eNB } \times f \\
& \text { INNC }=\text { INC }+ \text { F } \\
& \text { eNC }=\text { ZNC INNC }
\end{aligned}
$$

(c) Sensitivity analysis

During the calculation of sensitivity $S_{j}$, it is not necessary to derive the symbolic functions and make partial differentiation. The approximate formula is

$$
\frac{\partial E_{\text {oux }}}{\partial Y_{j}}=\frac{\Delta E_{\text {oux }}}{\Delta Y_{j}}
$$

The procedure of cost calculation in (b) can be used for sensitivity analysis and the algorithm is:

(1) Give an increment $\Delta Y_{j}=0.01$

(2) Substitute $Y_{j}+\Delta Y_{j}$ in $Y$-object

(3) Calculate $\mathbf{E}_{\text {nout }}$

(4) $\Delta \mathbf{E}_{\text {out }}=\mathbf{E}_{\text {nout }}-\mathbf{E}_{\text {out }}$

(5) $S_{j}=\Delta E_{\text {out }} / \Delta Y_{j}$

(d) Productivity analysis

The procedure of cost calculation in (b) can also be used for productivity analysis and the algorithm is:

(1) Set up Y-object using general technological coefficient value

(2) Calculate $E_{\text {gout }}$ using Algorithm (b)

(3) Set up Y-object using ideal technological coefficient value

(4) Calculate $E_{\text {iout }}$ using Algorithm (b)

(5) $P_{i}=\left(E_{\text {iout }} / E_{\text {gou }}\right) \times 100$

\subsection{Solutions}

In order to make the computer-aided computation, APL programming language has been chosen as a programming tool. A set of different functions has been developed for making the programming easy. The program list of analysis of material flow, and unit cost of output material with ideal and general technological value and productivity index are shown in Appendix. 


\section{Conclusions}

This paper has presented a new modeling and analysis approach to material flow and productivity by the use of Manufacturing Systems Theory. The general procedure by which the production system can be modeled and analyzed is: (1) to establish primitive systems; (2) to determine the behavior graph and topological object; (3) to select sources; and (4) to formulate and compute solutions via Roth's diagram. The case study has proved that MST can be fruitfully utilized for the purposes of costing and production planning of multi-stage manufacturing systems and it is useful in the practical implementation of this methodology to the modeling and analysis of manufacturing systems. According to author's knowledge, the modeling approach is more general, systematic, unified and effective than any other approach. We conclude that Manufacturing Systems Theory (MST) may be a most prospective approach to modeling and analysis of manufacturing and engineering systems.

\section{References}

(1) Bjørke, Ø., Towards a manufacturing systems theory - Applications so far, Proceedings of Conference Manufacturing International '88. Atlanta, Georgia. USA., 1990.

(2) Bjørke, Ø. and Wang, K., Manufacturing systems theory - Examples, Institute report, Department of production engineering, NTH, Trondheim, 1990.

(3) Bjørke, $\varnothing$. and Wang, K., Manufacturing systems theory, Lecture notes, Norwegian Institute of Technology, Trondheim, 1993.

(4) Buzacott, J. A., Prediction of efficiency of production systems with internal storage, International Journal of Production Research, Vol. 6, p. $173 \quad$, 1968.

(5) Davis, R. P., and Kennedy, Jr. W. J., Markovian modelling of manufacturing systems, International Journal of Production Research, Vol. 25, p. 337, 1987.

(6) Davis, R. P., and Miller, D. M., Analysis of the machine requirements problem through a parametric Markovian model. SME technical paper MS79-69, 1979.

(7) Dobois, D., and Stecke, K., Using petrinets to represent production process. Proceedings of 22nd IEEE conference on decision and control, December, 1983.

(8) Egbelu, P. J., Davis, R. P., Wysk, R. A., and Tanchoco, J. M. A.. An economic model for machining of cast parts, Journal of Manufacturing Systems, Vol.1, p. 207, 1982.

(9) Hubka, V. and Eder, W. E., Theory of Technical Systems - A total concept Theory for Engineering Design, Springer-Vorlage, Berlin, 1988.

(10) Keening, H. E., Toad, Y, and Kesavan, H. K., Analysis of Discrete Physical System, McGraw-Hill, New York, 1967.

(11) Pichler, F., and Diaz, R. M., (Eds.), Computer aided systems theory EUROCAST'93, Spring-Vorlage Berlin Heidelberg, 1994.

(12) Stack, K., and Solberg, J. J., The optionality of unbalancing both workloads and machine group sizes in closed queuing networks of multi-server queues, Operations Research, Vol 33, p. 882, 1985.

(13) Sushil, Singh, N. and Jain, B. K., A physical system theory approach to material flow and productivity analysis, Engineering Costs and Production Economics, Vol. 13, pp.207-215, 1988 
(14) Wang, K. and Bjørke, Ø., Manufacturing systems theory in Scandinavia, International symposium on manufacturing science \& technology for 21 st century, Beijing, 1994.

(15) Wang, K. and Bjørke, Ø., A general, efficient approach to dynamic analysis of interconnected mechanical system using the theory of connections, International journal of system science, Taylor \& Francis Ltd., Vol. 25, No. 7, pp. 1157-1178, 1994.

\section{Appendix}

1. Material flow with general technological coefficient value

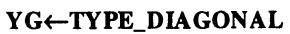

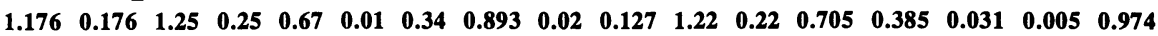
$\begin{array}{llllllllllll}0.001 & 0.053 & 1.053 & 0.32 & 0.01 & 0.69 & 1.54 & 0.54 & 0.28 & 0.22 & 0.94\end{array}$

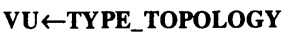

TYPE INCIDENCE PART OF BRANIN TABLE

$\begin{array}{lllllllllllllllllllllllllll}19 & 19 & 20 & 20 & 21 & 21 & 21 & 22 & 22 & 22 & 23 & 23 & 24 & 24 & 25 & 25 & 25 & 26 & 26 & 27 & 27 & 28 & 28 & 28 & 30 & 30 & 2\end{array}$ 29

$\begin{array}{lllllllllllllllllllllllllllll}1 & 2 & 19 & 3 & 20 & 4 & 5 & 21 & 6 & 7 & 22 & 8 & 23 & 25 & 9 & 10 & 26 & 11 & 27 & 12 & 28 & 30 & 15 & 29 & 13 & 14 & 16 & 17 & 18\end{array}$

TYPE TRANSFORMER PART OF BRANIN TABLE

YNG $\leftarrow \cdot$ (TRA VN) MUL YG MUL VP

YDIG $\leftarrow$ TYPE_DIAGONAL

$\begin{array}{llllllllllllllllllllllllllllll}1 & 1 & 1 & 1 & 1 & 1 & 1 & 1 & 1 & 1 & 1 & 1 & 1 & 1 & 1 & 1 & 1 & 1 & 1 & 1 & 1 & 1 & 1 & 1 & 1 & 1 & 1 & 1 & 1 & 1\end{array}$

YNNG $\leftarrow$ YNG + YDIG

ZNBG $\leftarrow$ INV YNNG

INB $\leftarrow \begin{array}{llllllllllllllllllllllllllllll}0 & 0 & 0 & 0 & 0 & 0 & 0 & 0 & 0 & 0 & 0 & 0 & 0 & 0 & 0 & 0 & 0 & 0 & 0 & 0 & 0 & 0 & 0 & 1 & 0 & 0 & 0 & 0 & 0 & 0\end{array}$

eNBG $\leftarrow Z N B G$ MUL INB

eNBG

$\begin{array}{llllllllllll}0.7565 & 0.1132 & 0.1287 & 0.007681 & 0.2611 & 0.172 & 0.1092 & 0.1551 & 0.01194 & 0.001925 & 0.000375 & 0.01989\end{array}$

$\begin{array}{llllllllllllll}0.1948 & 0.0683 & 0.003953 & 0.07636 & 0.06 & 0.2564 & 0.6433 & 0.5146 & 0.7681 & 0.8601 & 0.705 & 1 & 0.385 & 0.375\end{array}$

$\begin{array}{llll}0.3754 & 0.3953 & 0.2727 & 0.1265\end{array}$

(2) Unit cost of output material with general technological coefficient value

ZNCG $\leftarrow$ TRA ZNBG

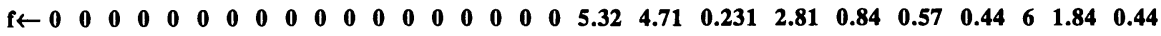

8.635 .46

FG $\leftarrow$ eNBGXf

$\begin{array}{llllllllllllllll}\text { INC } \leftarrow 1028 & -250 & -150 & -150 & 13632 & -10000 & 40972 & -200 & 113119 & -200 & -200 & -200 & 332 & 50 & -200 & 13667\end{array}$

$\begin{array}{rllllllllllllllll}-4000 & 13632 & 0 & 0 & 0 & 0 & 0 & 0 & 0 & 0 & 0 & 0 & 0 & 0\end{array}$

INNCG $\leftarrow$ INC + FG

eNCG $\leftarrow$ ZNCG MUL INNCG

eNCG

$\begin{array}{llllllllllllllll}1028 & -250 & -150 & -150 & 1.36 E 4 & -1.0 E 4 & 4.097 E 4 & -200 & 1.131 E 5 & -200 & -200 & -200 & 3323 & -150 & -200\end{array}$

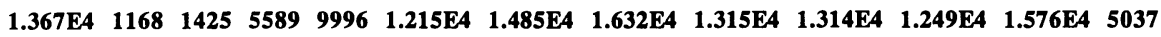

(3) Unit cost of outputmaterial for ideal technological coefficient value 


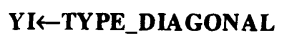

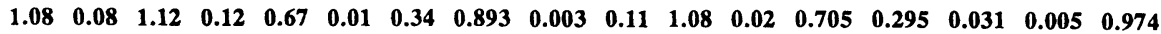
$\begin{array}{lllllllllllll}0.001 & 1.001 & 0.03 & 1.03 & 0.32 & 0.005 & 0.685 & 1.24 & 0.24 & 0.21 & 0.08 & 0.87\end{array}$

YNI - (TRA VN) MUL YI MUL VP

YNNI $\leftarrow$ YNI + YDIG

ZNBI $\leftarrow$ INV YNNI

eNBI $\leftarrow$ ZNBI MUL INB

ZNCI $\leftarrow$ TRA ZNBI

FI $\leftarrow$ eNBIxf

INNCI $\leftarrow$ ZNCI MUL INNCI

eNCI $\leftarrow$ ZNCI MUL INNCI

eNCI

$\begin{array}{llllllllllllllll}1028 & -250 & -150 & -150 & 1.363 E 4 & -1.0 E 4 & 4-09 E A & -200 & 1.131 E 5 & -200 & -200 & -200 & 3323 & -150 & -200 & 1.36 E 4\end{array}$

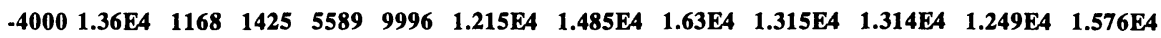
5037

(4) Computation of productivity index PI

$\square \leftarrow$ PROINDX $\leftarrow$ eNCI $\div$ eNCG $\times 100$

$\begin{array}{llllllllllllllllllll}100 & 100 & 100 & 100 & 100 & 100 & 100 & 100 & 100 & 100 & 100 & 100 & 100 & 100 & 100 & 100 & 100 & 100 & 93.55 & 84.77\end{array}$

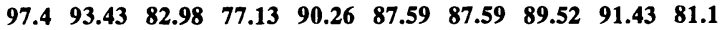

